\title{
Immunosuppression Induced by Perioperative Peritonitis Promotes Lung Metastasis
}

\author{
AKIRA ARIMOTO, KIMIHIRO YAMASHITA, HIROSHI HASEGAWA, YUTAKA SUGITA, \\ EIJI FUKUOKA, TOMOKO TANAKA, SATOSHI SUZUKI and YOSHIHIRO KAKEJI \\ Department of Surgery, Division of Gastrointestinal Surgery, \\ Kobe University Graduate School of Medicine, Kobe, Japan
}

\begin{abstract}
Background/Aim: Perioperative intra-abdominal infection has been reported as a risk factor for metastasis. The aim of this study was to investigate the mechanism by which peritonitis induces immunosuppression in the lung, which in turn promotes lung metastasis. Materials and Methods: C57BL/6 mice were intravenously administered B16F10 melanoma cells to induce lung metastasis and subsequently subjected to cecal ligation and puncture $(C L P)$ to induce peritonitis or sham surgery. The number of lung metastatic nodules was evaluated. Cell fractions in lungs and serum cytokines after CLP were investigated. Results: CLP mice showed an increased number of lung metastases compared to sham-treated mice. The fraction and number of natural killer (NK) cells in lungs of CLP mice were significantly reduced in early post-CLP phase. Myeloid-derived suppressor cells (MDSCs) in lungs were significantly decreased in CLP mice. Serum IL-6 and TNF levels were significantly elevated in CLP mice. Conclusion: Peritonitis promoted lung metastasis in a murine model, which may be attributable to the impact of NK cells and MDSCs in the lungs.
\end{abstract}

Perioperative intra-abdominal infection has been reported as a risk factor for systemic metastasis in patients with certain types of cancers (1-5). Some hypotheses have been proposed to explain the underlying mechanism for this association. One of the hypotheses is the typically severe inflammation associated with postoperative intra-abdominal infection switches off apoptosis, which shifts the balance in favor of tumor growth (6). However, there is no clear consensus on the mechanism by which postoperative infection leads to a poor prognosis.

Correspondence to: Kimihiro Yamashita, MD, Ph.D., Department of Surgery, Division of Gastrointestinal Surgery, Kobe University Graduate School of Medicine, 7-5-2 Kusunoki-cho, Chuo-ku, Kobe, Hyogo 650-0017, Japan. Tel: +81 783825925, Fax: +81783825939 , e-mail: kiyama@med.kobe-u.ac.jp

Key Words: Peritonitis, lung metastasis, NK cell, MDSCs.
The lung is the most frequent site of metastatic lesions in the context of several types of cancers, such as colorectal cancer, breast cancer and melanoma. Few studies have reported the impact of peritonitis on lung metastasis in a mouse model, although some experimental studies have investigated the influence of peritonitis on growth of subcutaneous tumors and liver metastasis $(7,8)$.

We hypothesized that peritonitis induces immunosuppression in the lung, which in turn promotes lung metastasis.

\section{Materials and Methods}

Mice and cell lines. Pathogen-free female C57BL/6J mice (5-8 weeks old) were purchased from CLEA Japan (Tokyo, Japan). All the mice were maintained under pathogen-free conditions and used in accordance with the institutional guidelines of Kobe University (approval number: P161002). The B16F10 murine melanoma cell line was purchased from American Type Culture Collection (Manassas, VA, USA) and were maintained in RPMI-1640 medium supplemented with $10 \%(\mathrm{v} / \mathrm{v})$ heat-inactivated fetal bovine serum, $2 \mathrm{mM}$ glutamine, $100 \mathrm{U} / \mathrm{ml}$ penicillin, and $100 \mu \mathrm{g} / \mathrm{ml}$ streptomycin (Sigma-Aldrich, St Lois, MO, USA) at $37^{\circ} \mathrm{C}$ in $5 \% \mathrm{CO}_{2}$. Cultured cells tested negative for mycoplasma and viral contamination.

Mouse model. To induce a mice model to form lung metastasis, mice were intravenously administered with $5 \times 10^{5}$ B16F10 melanoma cells. A model of peritonitis induced by cecal ligation and puncture (CLP) was used. The following operation was performed immediately after the injection of tumor cells. The mice were anaesthetized with intraperitoneal injection of a combination of medetomine hydrochloride, midazolam and butorphanol tartrate. The cecum was exposed, ligatured at its external half and punctured through with a 26-gauge needle. The incision was sutured in layers. The control mice were subjected to sham surgery (only laparotomy). The mice were administered intraperitoneal injection of meropenem every $12 \mathrm{~h}$ for 3 days.

Lung mononuclear cell and splenocyte isolation. Excised lung specimens were minced and suspended by using $1 \%$ collagenase type I (Wako Pure Chemical Industries, Osaka, Japan) in HBSS (ThermoFisher, Milan, Italy) at $37.0^{\circ} \mathrm{C}$ for $60 \mathrm{~min}$ for lung mononuclear cell (LMC) isolation. The cell suspension was passed through a $100-\mu \mathrm{m}$ cell strainer. The flow-through was suspended in 
33\% Percoll solution and centrifuged at $2000 \mathrm{rpm}$ for $20 \mathrm{~min}$ at room temperature. The spleen specimens were minced and passed through a $100-\mu \mathrm{m}$ cell strainer. Red blood cells were lysed with lysis buffer $\left(155 \mathrm{mM} \mathrm{NH} 4 \mathrm{Cl}\right.$ in $10 \mathrm{mM}$ Tris- $\mathrm{HCl}$ buffer, $\left.\mathrm{pH} 7.5,25^{\circ} \mathrm{C}\right)$ to obtain lung mononuclear cells and splenocytes. The cells were stained with the antibodies described below and used for flow cytometry.

Antibodies and flow cytometry. The following anti-mouse monoclonal antibodies were purchased from BD Biosciences (San Jose, CA, USA): anti-NK1.1; anti-CD11b; anti-CD11c; anti-Gr1; anti-Ly6C; anti-Ly6G; anti-CD3; and anti-CD19. Anti-CD45, anti-NKG2D, antiKLRG1, and anti-Sca1 were purchased from BioLegend (San Diego, CA, USA). Anti-CD69 was purchased from Thermo Fisher Scientific (Rockford, IL, USA). Flow cytometric data were obtained using a FACS Verse (Becton Dickinson, San Jose, CA, USA) and analyzed using FlowJo software (TreeStar, Ashland, OR, USA).

Cytokine. Serum cytokine levels were measured by cytometric bead array (CBA). The detailed procedure is described elsewhere (9). Briefly, plasma was collected from the peripheral blood of CLP mice and sham treated mice and aliquoted into polypropylene microcentrifuge tubes and stored at $-80^{\circ} \mathrm{C}$ until further processing. Concentrations of different cytokines were quantitatively measured using beads coated with antibodies against the following mouse cytokines: interleukin 6 (IL6), interleukin 10 (IL10), interleukin 12 p70 (IL12p70), Interferon $\gamma$ (IFN $\gamma$ ) and tumor necrosis factor (TNF). Stained samples were run on a FACS Verse (Becton Dickinson) instrument. All the samples were assayed in duplicate and were quantitated using a standardized curve. The data were analyzed using FACSArray (BD Biosciences, San Jose, CA, USA).

Statistics. Statistical analyses including Student's $t$-test and MannWhitney $U$-test were performed using JMP software (SAS Institute Inc., Cary, NC, USA). A value of $p<0.05$ was considered statistically significant.

\section{Results}

Lung metastasis in CLP mice. In order to evaluate the impact of peritonitis on lung metastasis, a double-hit model in which mice were intravenously injected with B16F10 melanoma cells was set up, followed by CLP or sham operation. In CLP mice, significantly larger number of lung metastatic nodules was observed as compared to that in sham treated mice (Figure 1).

Cell fraction assay in lung. To assess the effect of peritonitis on the immune system, the immune cell fraction and numbers in lung and spleen were compared between the CLP and sham treated mice (Figure 2). In CLP mice, the fraction and number of natural killer (NK) cells in the lung significantly reduced on day 1 . The difference persisted until day 8 . The proportion of myeloid-derived suppressor cells (MDSCs) in the lung of the CLP mice was significantly higher than that in the lung of the sham treated mice during the observation period. The CLP mice exhibited a slight increase in the number of MDSCs in the lung until day 4 , followed by a significant increase on day 8 . The proportions of CD4 and CD8 T cells in the lung were similar between the CLP and sham treated mice. The numbers of CD4
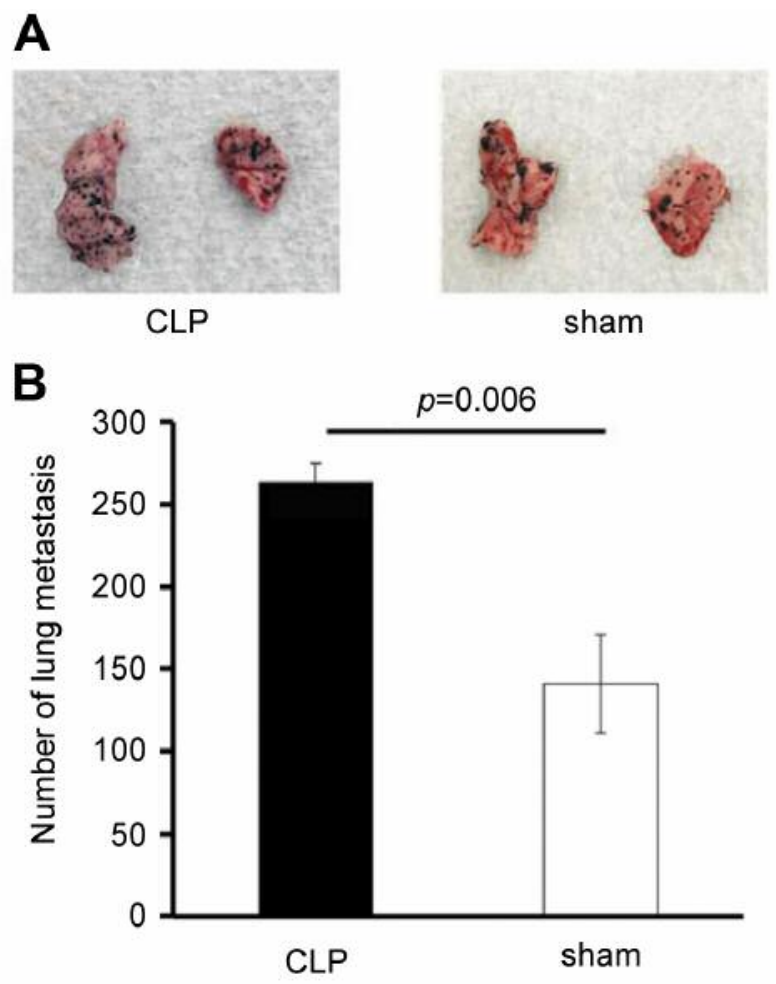

Figure 1. CLP promoted lung metastasis. C57BL/6 mice were injected intravenously with $5 \times 10^{5}$ B16F10 melanoma cells followed by CLP or sham surgery ( $n=5$, respectively). (A) Representative images of lung metastasis on day 14 after injection are shown. Black nodules indicate lung metastases. (B) The number of lung metastases significantly increased in CLP mice. CLP: Cecal ligation and puncture.

and CD8 T cells were significantly decreased in the CLP mice on day 1 but returned to a similar level after day 2 . The immune cells showed a similar tendency in both the lung and the spleen.

Activation and inhibition marker of $N K$ cells. To assess the impact of peritonitis on $\mathrm{NK}$ cell function in the lung, activation and inhibition of receptors on NK cells on day 1 after CLP were evaluated. The CLP mice exhibited significantly increased expressions of NKG2D and CD69, which are activation markers on NK cells (Figure 3 ). No significant difference was observed with respect to the other activation marker, Sca-1 and the inhibition marker, KLRG1. The numbers of NKG2D+, CD69+ and Sca-1+ NK cells in lung were similar in the CLP and sham treated mice. The number of KLRG1+ NK cells in CLP mice was significantly higher than that in sham treated mice.

Subsets of MDSCs in the lung. MDSCs are known to have at least two distinct subsets: granulocytic MDCSs (gMDSCs) and monocytic MDSCs (10). Therefore, the MDSC subsets were evaluated in detail (Figure 4). The proportion of 


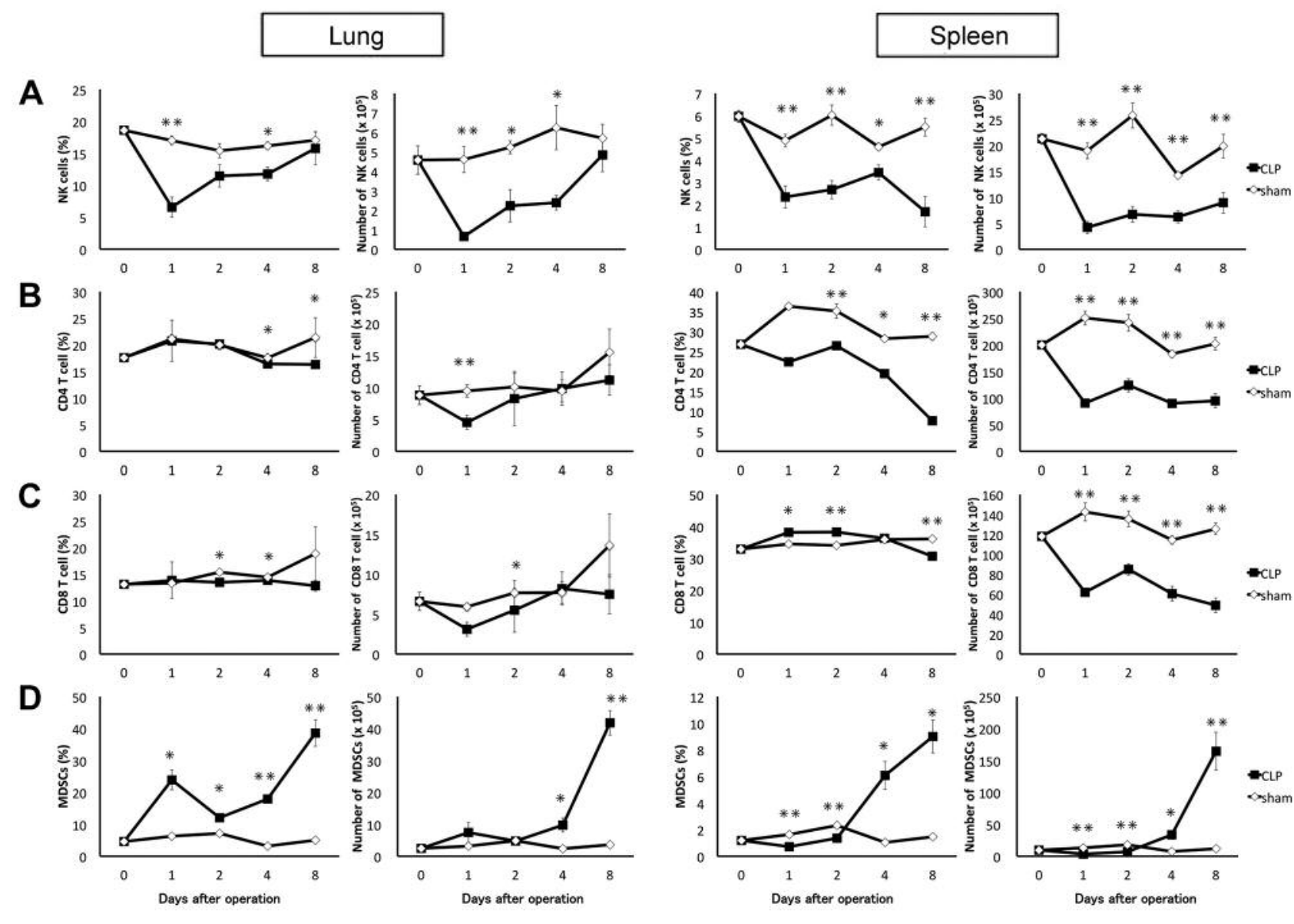

Figure 2. CLP induced immunosuppression in the lung and the spleen. Distribution and actual number of (A) NK cells, (B) CD4 T cells, (C) CD8 $T$ cells, and (D) MDSCs in the lung and the spleen of CLP and sham treated mice ( $n=4-5$, respectively) are shown. ${ }^{*} p<0.05,{ }^{* *} p<0.05 . C L P: C e c a l$ ligation and puncture; MDSCs: myeloid-derived suppressor cells.

gMDSCs was almost similar between the groups during the observation period with the exception of day 4 when the CLP mice showed a significantly higher proportion of gMDSCs as compared to that in sham treated mice.

Serum cytokines. To understand the mechanism by which peritonitis impaired the systemic immune response, the innate response of serum cytokines related with inflammation was monitored (Figure 5). In the CLP mice, serum levels of IL-6, IL-10 and TNF were significantly elevated on day 1 and rapidly decreased thereafter. In contrast, the serum levels of IL12p70 and IFN- $\gamma$ were not significantly different between the CLP and sham treated mice.

\section{Discussion}

Patients who suffer from postoperative infection were shown to be at a higher risk of death from metastasis compared to patients who did not (11). Experimental evidence suggests that abdominal infection induces systemic infection following the formation of a microenvironment advantageous for tumor growth $(8,12)$. In the present study, we hypothesized that peritonitis induces immunosuppression in the lung, which in turn promotes lung metastasis. Supporting this hypothesis, the CLP mice showed promotion of lung metastasis. The CLP mice also exhibited a quantitative reduction of NK cells and increase in MDSCs in the lung. Furthermore, the CLP mice showed high serum levels of IL-6 and TNF, known to suppress NK cell function.

In anticancer immunity, NK cells play an important role in the immune system, particularly the innate immune system. Previous studies reported that NK cell depletion by antiASGM1 antibody or anti-NK1.1 mAb, but not CD4 nor CD8 $\mathrm{T}$ cell depletion, significantly augmented B16 lung metastasis in WT mice $(13,14)$. In the present study, the CLP mice showed significant reduction in NK cells in lungs on day 1 (Figure 2), which is one of the potential mechanisms for promotion of lung metastasis in the CLP mice. 

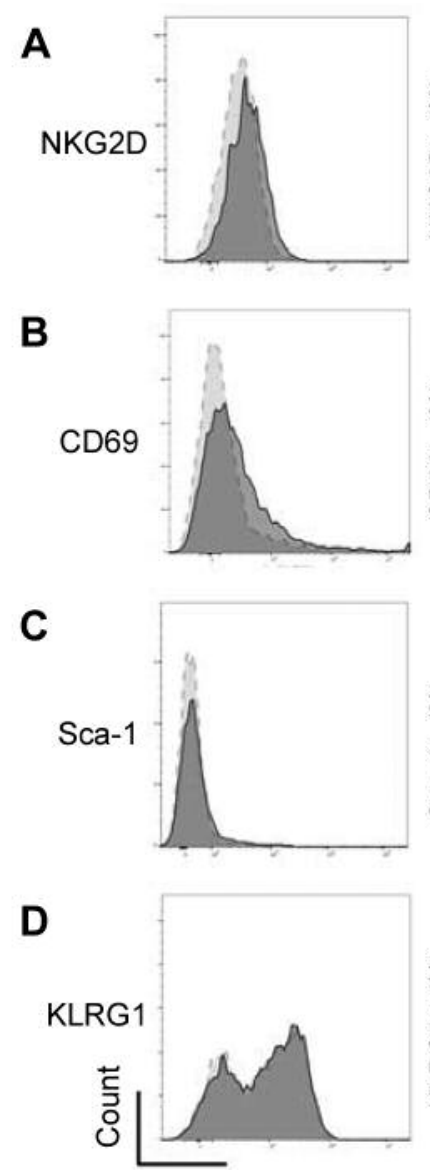

Fluorescence intensity
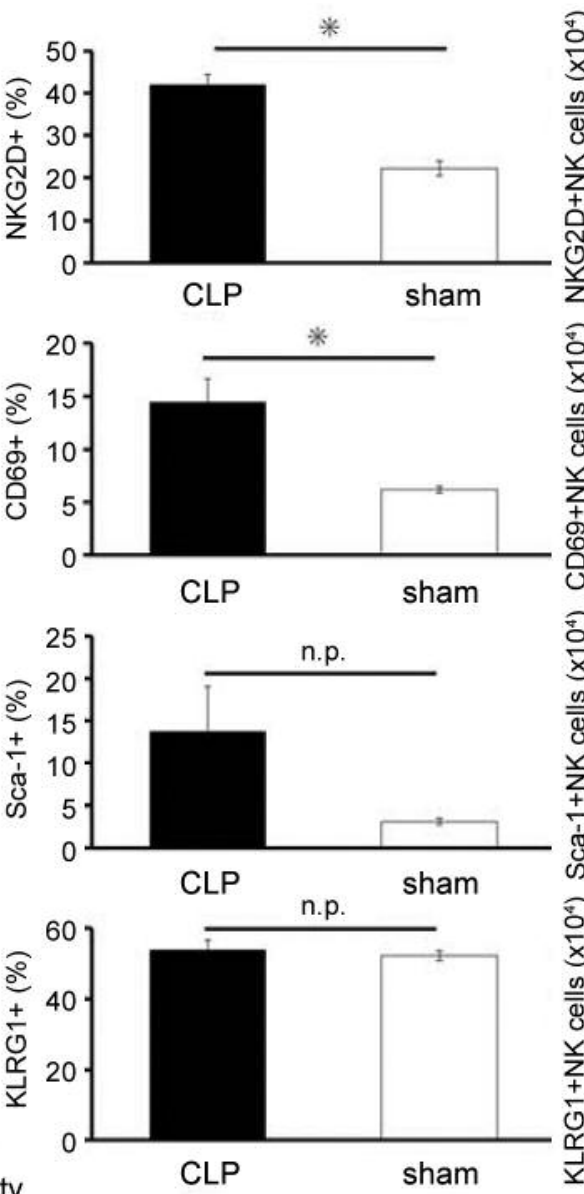
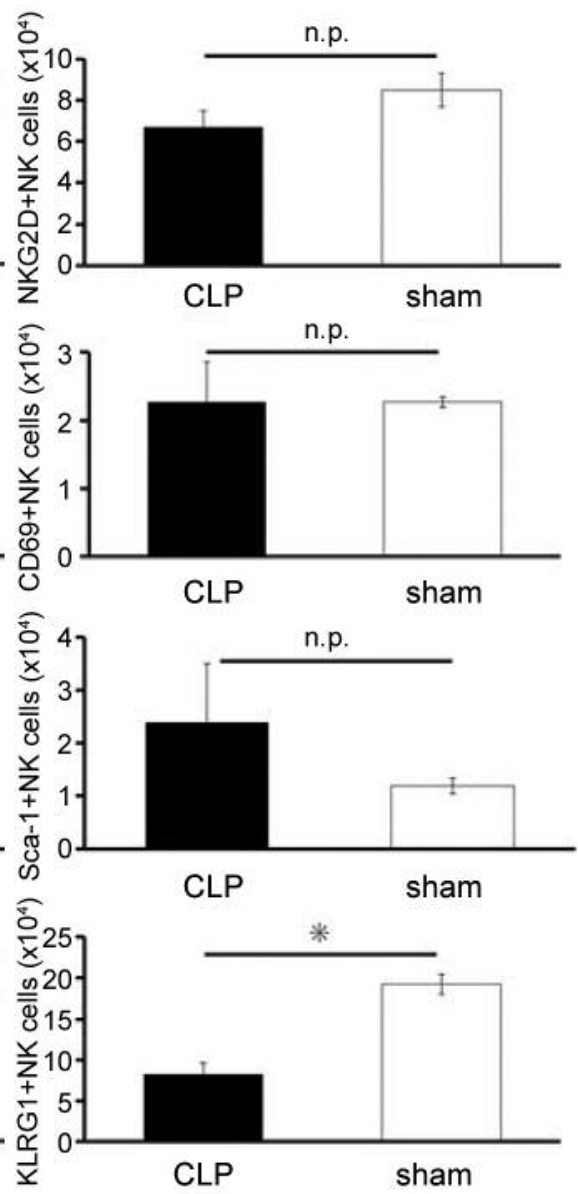

Figure 3. The number of activated NK cells in the lung was similar between CLP and sham-treated mice. CLP mice exhibited-significantly increased frequency of (A) NKG2D and (B) CD69 expression (activation markers on NK cells). Other activation marker, (C) Sca-1, and inhibition marker, (D) KLRG1, did not show significant difference. The numbers of NKG2D+, CD69+, and Sca-1+NK cells in the lung were similar between CLP and sham-treated mice $(n=4$, respectively). Solid line histogram and dotted line histogram show cells in CLP and sham treated mice, respectively. $* p<0.05$. CLP: Cecal ligation and puncture.

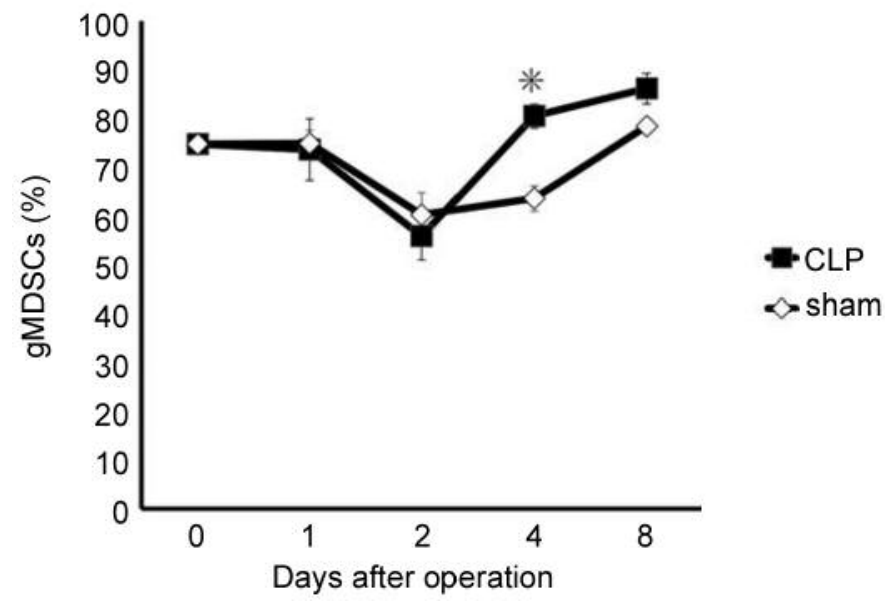

Figure 4. Proportion of granulocytic myeloid-derived suppressor cells (MDSCs) preferentially increased in CLP mice. MDSC subsets in the lung were

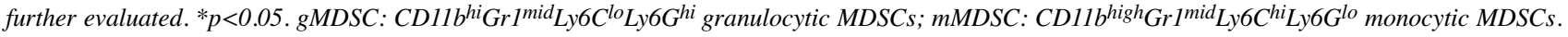



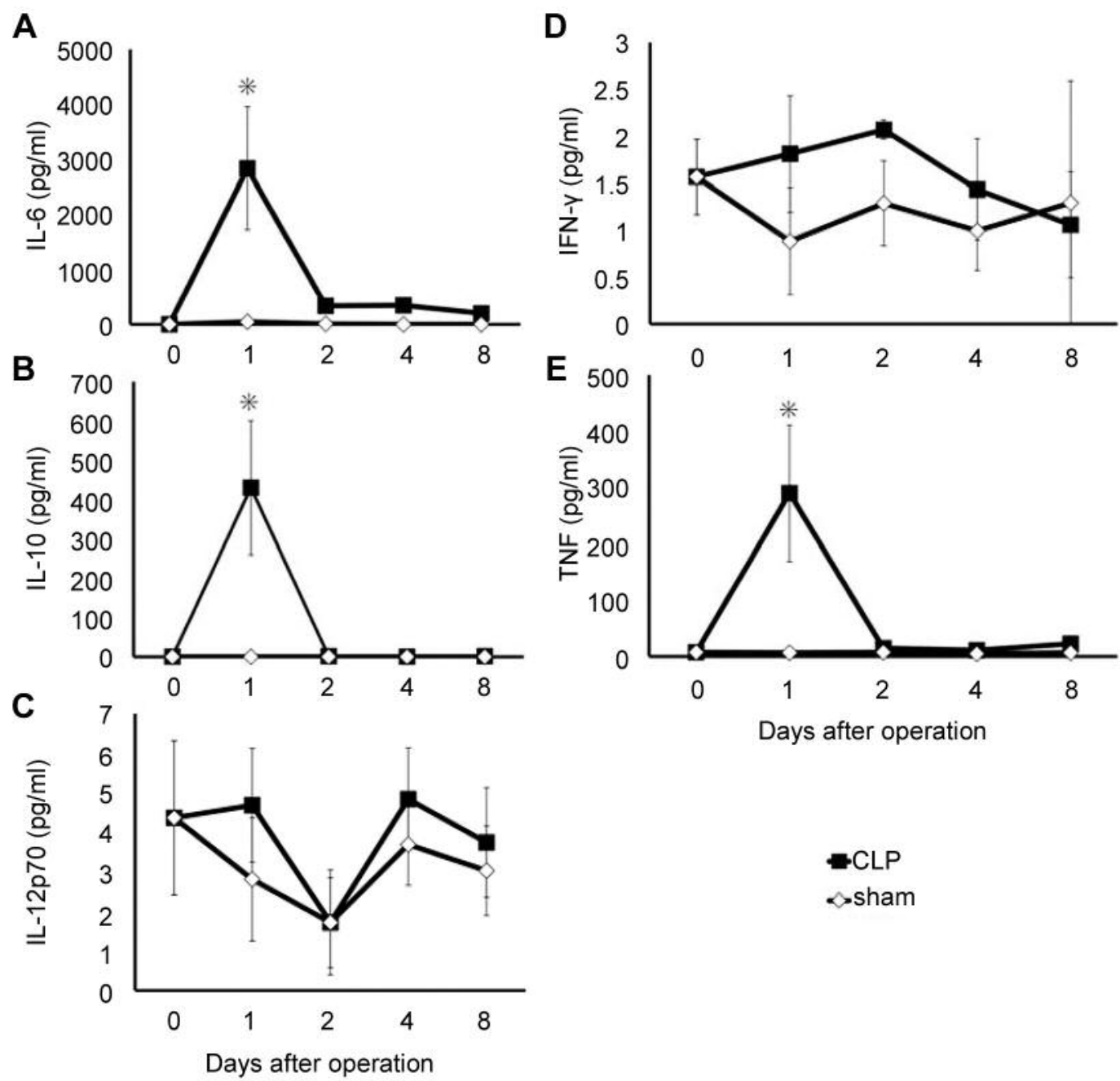

Figure 5. Elevation of serum cytokines suggested a suppression of NK cell activity. Plasma was collected from the peripheral blood of CLP mice and sham-treated mice ( $n=4-5$, respectively). The following cytokines were measured by cytometric bead array (CBA) and flow cytometry: interleukin 6 (IL6), interleukin 10 (IL10), interleukin 12p70 (IL12p70), interferon $\gamma($ IFN $\gamma)$ and tumor necrosis factor (TNF). *p<0.05. CLP: Cecal ligation and puncture.

Multiple activation and inhibitory receptors regulate the NK cell function (15). Expressions of NKG2D and CD69 (activation markers of NK cells) in the CLP mice were higher than that in sham treated mice on day 1 (Figure 3 ). The number of activated the NK cells in the lung was comparable in the two groups. However, no significant between-group difference was observed with respect to cytotoxicity using LMC (data not shown). Although killer cell lectin-like receptor G1 (KLRG1) is known as an inhibitory receptor on NK cells, Huntington et al. reported that KLRG1 expression is related to continuous $\mathrm{NK}$ cell maturation in the periphery (16). The number of KLRG1+ NK cells may be associated with prolonged NK cell activation.

Llitjos et al. reported that sepsis-induced gMDSCs promote tumor growth (7). In the present study, MDSCs in the lung and spleen were significantly increased in the CLP mice on day 8 after operation (Figure 2). Subset analysis of MDSCs showed similar proportion of gMDSCs between the CLP and sham treated mice during the observation period with the exception of day 4 (Figure 4). The accumulation of gMDSCs in the lung may render the microenvironment favorable for tumor growth.

Lung metastasis seems to be regulated by both local and systemic antitumor response in the CLP mice inoculated with tumor cells. It is well known that microbial infection can induce systemic inflammation via both pro- and antiinflammatory mediators. High serum levels of inflammatory cytokines are believed to accelerate tumor metastasis via promotion of growth factors or suppression of host immune response against tumors. In the CLP mice, levels of IL-6, IL- 
10 , and TNF showed a remarkable increase in the early phase after CLP (Figure 5). Increased level of IL-6 was shown to suppress NK cell activity via down-regulation of cytotoxic granule components through modulation of the expression of Src homology region 2-containing protein tyrosine phosphatase-2 (SHP-2) (17). It is also well known that TNF promotes metastasis by impairing NK cell activity (18). The significant elevation of cytokine levels is believed to suppress NK cell activity in the lungs. Our data indicate an important contribution of NK cells in the suppression of lung metastasis. This finding has significant implications since we reported previously that dendritic cells, pulsed with the glycolipid $\alpha$ galactosylceramide ( $\alpha$-GalCer), induce activation of invariant NKT cells following activation of the NK cells in the lung (19). The production of IFN- $\gamma$ by the subsequently activated NK cells was associated with an anti-metastatic activity. The NK cell activation by dendritic cells pulsed with $\alpha$-GalCer appears to be a feasible strategy to suppress lung metastasis in patients with perioperative peritonitis.

In conclusion, peritonitis can promote lung metastasis in a murine lung metastasis model, an effect that is possibly mediated by NK cell reduction and MDCSs accumulation in the lung.

\section{Conflicts of Interest}

None of the Authors have any conflict of interest with respect to this study.

\section{Acknowledgements}

This work was supported by a Grant-in-Aid from the Ministry of Education, Culture, Sports, Science, and Technology of Japan (AA: No. 17K16554; YK: No. 16H05415).

\section{References}

1 Walker KG, Bell SW, Rickard MJ, Mehanna D, Dent OF, Chapuis PH and Bokey EL: Anastomotic leakage is predictive of diminished survival after potentially curative resection for colorectal cancer. Ann Surg 240: 255-259, 2004.

2 McArdle CS, McMillan DC and Hole DJ: Impact of anastomotic leakage on long-term survival of patients undergoing curative resection for colorectal cancer. Br J Surg 92: 1150-1154, 2005.

3 Bohle B, Pera M, Pascual M, Alonso S, Mayol X, Salvado M, Schmidt J and Grande L: Postoperative intra-abdominal infection increases angiogenesis and tumor recurrence after surgical excision of colon cancer in mice. Surgery 147: 120-126, 2010.

4 Tam V, Luketich JD, Winger DG, Sarkaria IS, Levy RM, Christie NA, Awais O, Shende MR and Nason KS: Cancer recurrence after esophagectomy: impact of postoperative infection in propensitymatched cohorts. Ann Thorac Surg 102: 1638-1646, 2016.

5 Tsujimoto H, Ichikura T, Ono S, Sugasawa H, Hiraki S, Sakamoto N, Yaguchi Y, Yoshida K, Matsumoto Y and Hase K: Impact of postoperative infection on long-term survival after potentially curative resection for gastric cancer. Ann Surg 16: 311-318, 2009.
6 Bell SW, Walker KG, Rickard MJFX, Sinclair G, Dent OF, Chapuis PH and Bokey EL: Anastomotic leakage after curative anterior resection results in a higher prevalence of local recurrence. Br J Surg 90: 1261-1266, 2003.

7 Llitjos JF, Auffray C, Alby-Laurent F, Rousseau C, Merdji H, Bonilla N, Toubiana J, Belaïdouni N, Mira JP, Lucas B, Chiche JD and Pène F: Sepsis-induced expansion of granulocytic myeloid-derived suppressor cells promotes tumor growth through Toll-like receptor 4. J Pathol 239: 473-483, 2016.

8 Matsumoto Y, Tsujimoto H, Ono S, Shinomiya N, Miyazaki H, Hiraki S, Takahata R, Yoshida K, Saitoh D, Yamori T, Yamamoto $\mathrm{J}$ and Hase $\mathrm{K}$ : Abdominal infection suppresses the number and activity of intrahepatic natural killer cells and promotes tumor growth in a murine liver metastasis model. Ann Surg Oncol 23: 257-265, 2016.

9 Tanaka T, Fujita M, Hasegawa H, Arimoto A, Nishi M, Fukuoka E, Sugita Y, Matsuda T, Sumi Y, Kakeji Y and Yamashita K: Frequency of myeloid-derived suppressor cells in the peripheral blood reflects the status of tumor recurrence. Anticancer Res 37: 3863-3869, 2017.

10 Kumar V and Gabrilovich DI: Hypoxia-inducible factors in regulation of immune responses in tumor microenvironment. Immunology 143: 512-519, 2014.

11 Andalib A, Ramana-Kumar AV, Bartlett G, Franco EL and Ferri LE: Influence of postoperative infectious complications on longterm survival of lung cancer patients: a population-based cohort study. J Thorac Oncol 8: 554-561, 2013.

12 Deng JC, Cheng G, Newstead MW, Zeng X, Kobayashi K, Flavell RA and Standiford TJ: Sepsis-induced suppression of lung innate immunity is mediated by IRAK-M. J Clin Invest 116: 2532-2542, 2006.

13 Takeda K, Nakayama M, Sakaki M, Hayakawa Y, Imawari M, Ogasawara K, Okumura K and Smyth MJ: IFN- $\gamma$ production by lung NK cells is critical for the natural resistance to pulmonary metastasis of B16 melanoma in mice. J Leukoc Biol 90: 777785, 2011.

14 Park JM, Terabe M, Van Den Broeke LT, Donaldson DD and Berzofsky JA: Unmasking immunosurveillance against a syngeneic colon cancer by elimination of CD4+NKT regulatory cells and IL-13. Int J Cancer 114: 80-87, 2005.

15 Fogel LA, Sun MM, Geurs TL, Carayannopoulos LN and French AR: Markers of nonselective and specific NK cell activation. J Immunol 190: 6269-6276, 2013.

16 Huntington ND, Tabarias H, Fairfax K, Brady J, Hayakawa Y, Degli-Esposti MA, Smyth MJ, Tarlinton DM and Nutt SL: NK cell maturation and peripheral homeostasis is associated with KLRG1 up-regulation. J Immunol 178: 4764-4770, 2007.

17 Kang YJ, Jeung IC, Park A, Park YJ, Jung H, Kim TD, Lee HG, Choi I and Yoon SR: An increased level of IL-6 suppresses NK cell activity in peritoneal fluid of patients with endometriosis via regulation of SHP-2 expression. Hum Reprod 29: 2176-2189, 2014.

18 Hafner $M$ and Orosz P: TNF promotes metastasis by impairing natural killer cell activity. Int J Cancer 66: 388-392, 1996.

19 Hasegawa H, Yamashita K, Otubo D, Fujii SI, Kamigaki T, Kuroda D and Kakeji Y: Allogeneic DCG promote lung NK cell activation and antitumor effect after invariant NKT cell activation. Anticancer Res 34: 3411-3417, 2014.

Received April 11, 2018

Revised May 11, 2018

Accepted May 14, 2018 